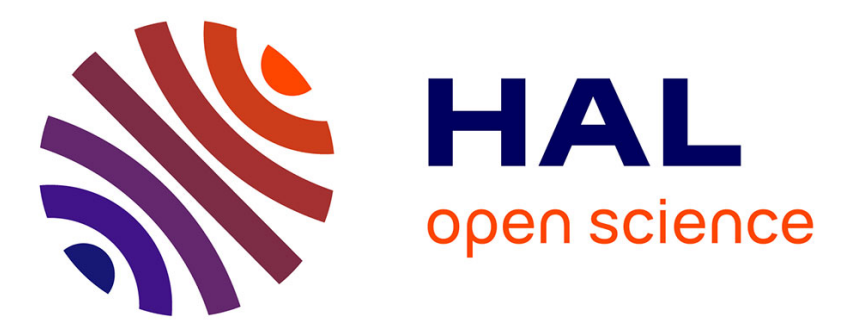

\title{
On the Dependence of the Electron Paramagnetic Resonance Line Intensities on the Microwave Field Orientation
}

Kira Seleznyova, Sergey Yagupov, Mark Strugatsky, Janis Kliava

\section{To cite this version:}

Kira Seleznyova, Sergey Yagupov, Mark Strugatsky, Janis Kliava. On the Dependence of the Electron Paramagnetic Resonance Line Intensities on the Microwave Field Orientation. Applied Magnetic Resonance, 2015, 46 (11), pp.1323-1330. 10.1007/s00723-015-0723-y · hal-01229373

\section{HAL Id: hal-01229373 \\ https://hal.science/hal-01229373}

Submitted on 16 Nov 2015

HAL is a multi-disciplinary open access archive for the deposit and dissemination of scientific research documents, whether they are published or not. The documents may come from teaching and research institutions in France or abroad, or from public or private research centers.
L'archive ouverte pluridisciplinaire HAL, est destinée au dépôt et à la diffusion de documents scientifiques de niveau recherche, publiés ou non, émanant des établissements d'enseignement et de recherche français ou étrangers, des laboratoires publics ou privés.

\section{다(1)(2)}

Distributed under a Creative Commons Attribution - ShareAlikel 4.0 International 


\title{
On the Dependence of the Electron Paramagnetic Resonance Line Intensities on the Microwave Field Orientation
}

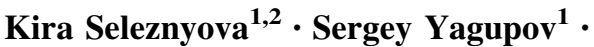 \\ Mark Strugatsky ${ }^{1} \cdot$ Janis Kliava $^{2}$
}

\begin{abstract}
Electron paramagnetic resonance (EPR) spectra of Ni-doped gallium borate show a surprisingly pronounced dependence of certain line intensities on the orientation of the microwave field $\boldsymbol{B}_{1}$ with respect to the crystal axes. In order to account for this dependence, we have considered in detail the $\boldsymbol{B}_{1}$ caused perturbation matrix and obtained an expression of the angular dependence of transition probabilities between spin levels. Computer-generated EPR spectra taking into account this dependence provide good fits to the experimental spectra characteristics.
\end{abstract}

\section{Introduction}

In an electron paramagnetic resonance (EPR) experiment, the sample containing paramagnetic species is subjected to two different magnetic fields: the magnetizing ("static") field $\boldsymbol{B}$ and the magnetic component of the microwave field $\boldsymbol{B}_{1}$ of frequency $v$. As far as, in most cases of interest, $|\boldsymbol{B}|=\boldsymbol{B} \gg\left|\boldsymbol{B}_{1}\right|=B_{1}$, the role of the former is to split the ground state of the paramagnetic centers into Zeeman sublevels and that of the latter is to induce transitions between them. Sweeping $B$ produces the EPR spectra where different lines arise from transitions between different Zeeman sublevels, so that the positions of these lines are determined by the resonance condition (quantum energy = level splitting). In turn, the intensities of the spectra lines are determined by the transition matrix element that depends on the orientations of both $\boldsymbol{B}$ and $\boldsymbol{B}_{1}$. The implications of these dependences are particularly important in the EPR spectroscopy of single crystals, in which case, to

Janis Kliava

janis.kliava@u bordeaux.fr

1 Faculty of Physics and Computer Sciences, Crimean Federal University, Simferopol, Crimea, Russian Federation

2 LOMA, UMR 5798 Université de Bordeaux CNRS, 33405 Talence Cedex, France 
get good computer fits to the experimental EPR spectra, they should be carefully taken into account in the simulation codes. Meanwhile, in papers dealing with the EPR spectroscopy of single crystals, the effects of changing the orientation of $\boldsymbol{B}_{1}$ with respect of the crystallographic axes have only occasionally been analyzed, e.g., see [1].

The aim of the present paper is to provide a detailed description of the relation between the orientation of $\boldsymbol{B}_{1}$ and the EPR line intensities. The importance of taking into account this relation is illustrated by computer simulations of $\mathrm{Ni}^{3+} \mathrm{EPR}$ spectra in $\mathrm{GaBO}_{3}$ crystals.

\section{Theoretical Background}

If only a linear coupling between the (effective) electron spin $\boldsymbol{S}$ and the field $\boldsymbol{B}$ (Zeeman interaction) is taken into account, the spin Hamiltonian of a paramagnetic center can be expressed as follows [2]:

$$
H=\beta B \cdot \boldsymbol{g} \cdot S+(\text { crystal field terms })
$$

where $g$ is the electron $g$ tensor and $\beta$ is the Bohr magneton. The eigenvalues $E_{p}$ of the $H$ matrix are the energies of different spin states, while its eigenvectors $\Psi_{p}$ are the corresponding wave functions. Transitions between different spin states are of magnetic dipolar nature; therefore, they are induced by the magnetic component $\boldsymbol{B}_{1}$ of the electromagnetic wave. The matrix element of the perturbation operator $H_{1}$ due to the presence of the microwave magnetic field, between $p$ and $q$ spin states is $[2,3]$ :

$$
\mu_{p q}=\beta\left\langle\Psi_{p}\left|H_{1}\right| \Psi_{q}\right\rangle .
$$

The transition intensity, proportional to the number of transitions per unit time between the $p$ and $q$ states can be expressed as [2]:

$$
W_{p q} \propto v^{2}\left|\mu_{p q}\right|^{2}
$$

where $v$ is the frequency of $\boldsymbol{B}_{1}$. Thus, $W_{p q}$ depends on the orientations of both $\boldsymbol{B}$ (through $\Psi_{p}$ and $\Psi_{q}$ ) and $\boldsymbol{B}_{1}$ (explicitly). In the case of the spin Hamiltonian Eq. (1), this perturbation can be considered as the result of interaction between $\boldsymbol{B}_{1}$ and the electron magnetic moment with components [4]:

$$
(\beta g S)_{i}=\beta \sum_{i} g_{i} S_{i}, \quad i=x, y, z
$$

$x, y$ and $z$ being local magnetic axes. Thus,

$$
\mu_{p q}=\beta\left\langle\Psi_{p}\left|B_{1} \cdot g \cdot S\right| \Psi_{q}\right\rangle
$$

Let us assume for simplicity that the $x, y, z$ axis frame of the spin Hamiltonian Eq. (1) coincides with the crystallographic axis frame $x_{\mathrm{c}}, y_{\mathrm{c}}, z_{\mathrm{c}}$. We consider the laboratory axis frame $x_{1}, y_{1}, z_{1}$, defined by the configuration of the microwave cavity and its disposition with respect to the poles of the electromagnet of the EPR 
spectrometer. In most commercial EPR spectrometers the two magnetic fields are orthogonal, $\boldsymbol{B}_{1} \perp \boldsymbol{B}$. In what follows, both this condition and that of smallness of $B_{1}$ in comparison to $B$, see Sect. 1, are assumed to be satisfied. We choose the directions of $\boldsymbol{B}$ and $\boldsymbol{B}_{1}$ along $z_{1}$ and $y_{1}$, respectively. Relative orientations of the laboratory frame and the crystallographic one can be described by the following matrix [5]:

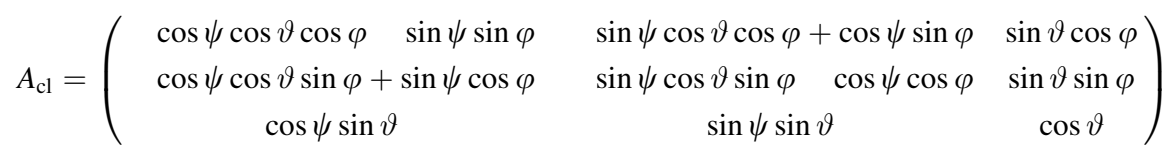

where $\vartheta, \varphi$ and $\pi-\psi$ are the Euler angles shown in Fig. 1. One can see that with such a definition, in the $x_{\mathrm{c}}, y_{\mathrm{c}}, z_{\mathrm{c}}$ frame $\vartheta$ and $\varphi$ are spherical angles of $\boldsymbol{B}$, and $\psi$ describes the orientation of $\boldsymbol{B}_{1}$ in the plane perpendicular to $\boldsymbol{B}$.

Thus, the unit vectors of $\boldsymbol{B}$ and $\boldsymbol{B}_{1}$ in the laboratory frame are:

$$
l_{l}=\left(\begin{array}{l}
0 \\
0 \\
1
\end{array}\right) \quad \text { and } \quad l_{l \ell}=\left(\begin{array}{l}
0 \\
1 \\
0
\end{array}\right)
$$

and in the crystallographic frame they become:

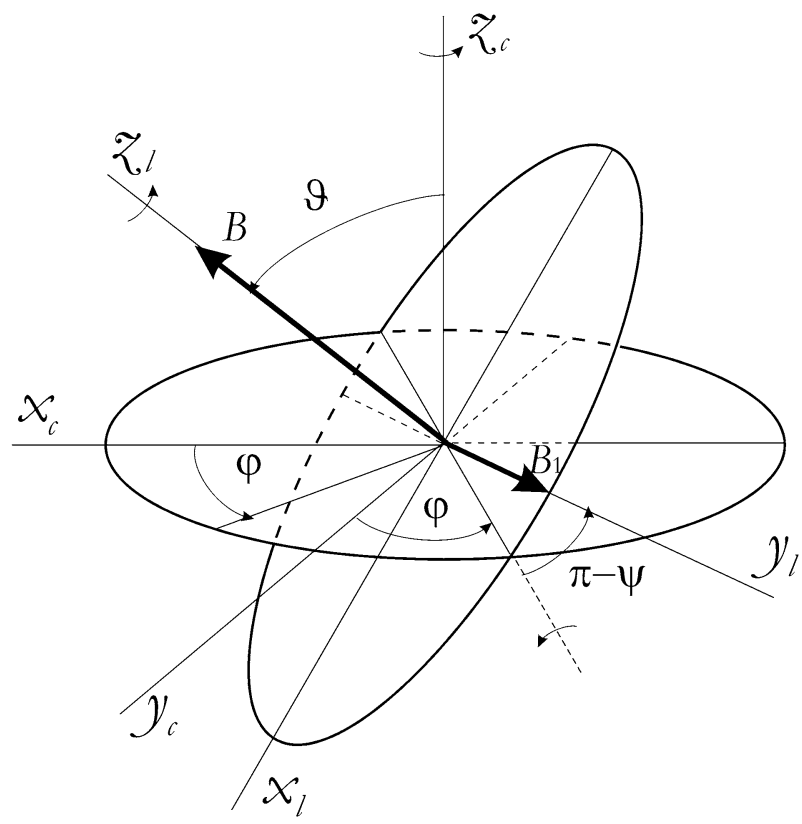

Fig. 1 Euler angles between the crystallographic frame $x_{\mathrm{c}}, y_{\mathrm{c}}, z_{\mathrm{c}}$ and the laboratory frame $x_{1}, y_{1}, z_{1}$ 


$$
\begin{gathered}
l=A_{\mathrm{cl}} l_{1}=\left(\begin{array}{c}
\sin \vartheta \cos \varphi \\
\sin \vartheta \sin \varphi \\
\cos \vartheta
\end{array}\right) \text { and } \\
l_{1}=A_{\mathrm{cl}} l_{1 l}=\left(\begin{array}{c}
-\sin \psi \cos \vartheta \cos \varphi+\cos \psi \sin \varphi \\
-\sin \psi \cos \vartheta \sin \varphi-\cos \psi \cos \varphi \\
\sin \psi \sin \vartheta
\end{array}\right) .
\end{gathered}
$$

Recently, we have studied EPR of $\mathrm{Fe}^{3+}(S=3 / 2)$ in $\mathrm{GaBO}_{3}$ [6]. In this case local symmetry of the paramagnetic ions is trigonal, so that Eq. (5) becomes:

$$
\mu_{p q}=\beta B_{1}\left\langle\Psi_{p}(\vartheta, \varphi)\left|g_{\|} l_{1 z} O_{1}^{0}+g_{\perp}\left(l_{1 x} O_{1}^{1}+l_{1 y} O_{1}^{1}\right)\right| \Psi_{q}(\vartheta, \varphi)\right\rangle
$$

where $O_{n}^{ \pm m}$ are extended Stevens operators (in Ref. [7] $O_{n}^{-m}$ are denoted as $\Omega_{n}^{m}$ ), $g_{\perp}=g_{x}=g_{y}$ and $g_{\|}=g_{z}$ are components of the $g$ tensor, and $l_{1 x}=-\sin \psi \cos \vartheta$ $\cos \varphi+\cos \psi \sin \varphi, l_{1 y}=-\sin \psi \cos \vartheta \sin \varphi-\cos \psi \cos \varphi$ and $l_{1 z}=\sin \psi \sin \vartheta$ are direction cosines of $\boldsymbol{B}_{1}$, see Eq. (8). Using Eq. (9) in computer simulations of the EPR spectra of $\mathrm{Fe}^{3+}$ in $\mathrm{GaBO}_{3}$ has provided close fits to the experimental EPR spectra [6].

By analogy, for $\mathrm{Ni}^{3+}(S=3 / 2)$ in $\mathrm{GaBO}_{3}$ we can expect the same type of symmetry. Meanwhile, we have found that to satisfactorily account for experimental EPR spectra of this ion, third-power terms in the electron spin should be taken into account in the Zeeman interaction. Thus, we have considered the spin Hamiltonian for trigonal symmetry given in the Al'tshuler's and Kozyrev's textbook [7] with some supplementary terms omitted therein:

$$
\begin{aligned}
H= & g_{\|} \beta B_{z} O_{1}^{0}+g_{\perp} \beta\left(B_{x} O_{1}^{1}+B_{y} O_{1}{ }^{1}\right)+B_{2}^{0} O_{2}^{0}+\beta B_{z}\left(g_{1} O_{3}^{0}+g_{2} O_{3}{ }^{3}+g_{3} O_{3}^{3}\right) \\
& +g_{4} \beta\left(B_{x} O_{3}^{1}+B_{y} O_{3}{ }^{1}\right)+g_{5} \beta\left(B_{x} O_{3}^{2}-B_{y} O_{3}{ }^{2}\right)+g_{6} \beta\left(B_{x} O_{3}{ }^{2}+B_{y} O_{3}^{2}\right)
\end{aligned}
$$

where $B_{2}^{0}$ is the quadrupole fine-structure parameter and $g_{1}, \ldots, g_{6}$ are parameters of different Zeeman terms in $S^{3}$. Meanwhile, such terms should also be taken into account in the perturbation operator; so, instead of Eq. (9) we get:

$$
\mu_{p q}=\beta B_{1}\left\langle\Psi_{p}(\vartheta, \varphi)\left|\begin{array}{ll}
g_{\|} l_{1 z} O_{1}^{0}+g_{\perp}\left(l_{1 x} O_{1}^{1}+l_{1 y} O_{1}^{-1}\right)+l_{1 z}\left(g_{1} O_{3}^{0}+g_{2} O_{3}^{-3}+g_{3} O_{3}^{3}\right) \\
+l_{1 x}\left(g_{4} O_{3}^{1}+g_{5} O_{3}^{2}+g_{6} O_{3}^{-2}\right)+l_{1 y}\left(g_{4} O_{3}^{-1}\right. & \left.g_{5} O_{3}^{-2}+g_{6} O_{3}^{2}\right)
\end{array}\right| \Psi_{q}(\vartheta, \varphi)\right\rangle .
$$

\section{Results and Discussion}

Gallium borate single crystal doped with nickel ( $0.26 \%$ of $\mathrm{Ni}$ with respect to $\mathrm{Ga})$ were prepared in the Crystal Growth Laboratory at the Simferopol University [8]. $\mathrm{GaBO}_{3}$ has rhombohedral calcite structure with the space group $\mathrm{D}_{3 \mathrm{~d}}^{6}$ [9]. The crystals were grown in the shape of thin hexagonal plates making 0.1 to $0.2 \mathrm{~mm}$ along the trigonal $\mathrm{C}_{3}$ axis and up to $5 \mathrm{~mm}$ in the basal plane perpendicular to the $\mathrm{C}_{3}$ axis.

The crystals were studied with an X-band $(9.464 \mathrm{GHz})$ spectrometer (Bruker) at $4 \mathrm{~K}$ in static magnetic fields up to $1 \mathrm{~T}$. The EPR spectra were measured in two 

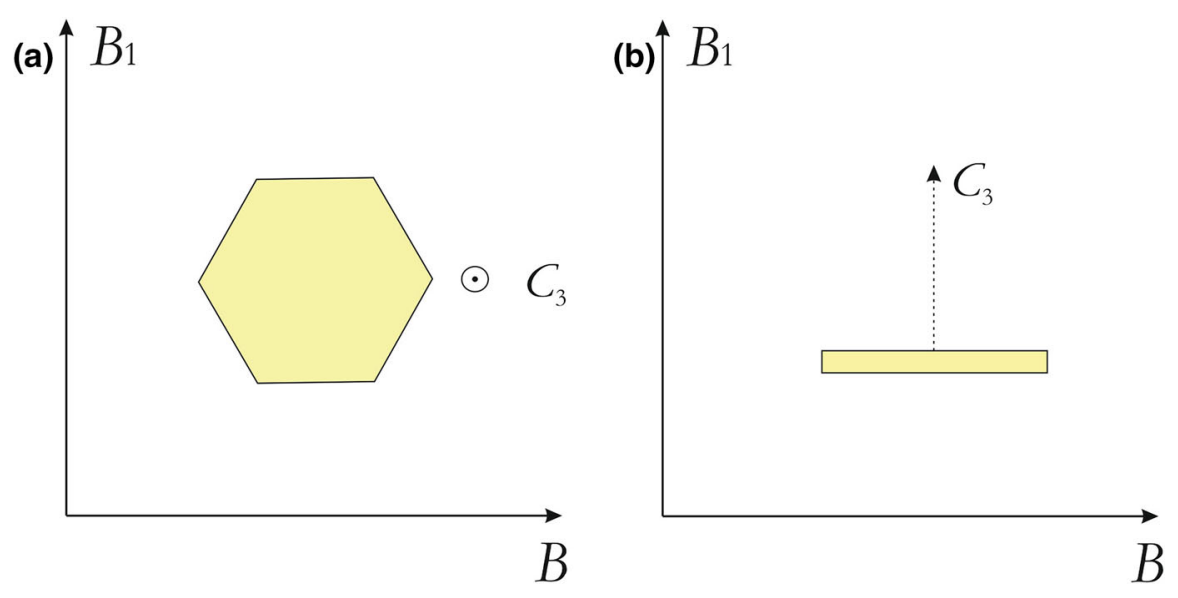

Fig. 2 Two different orientations of the sample with respect to the magnetic fields: $\mathbf{a} \boldsymbol{B}_{1} \perp \mathrm{C}_{3}$ and b $\boldsymbol{B}_{1} \| \mathrm{C}_{3}$. In both cases, $\boldsymbol{B} \perp \boldsymbol{B}_{1}$ and $\boldsymbol{B} \perp \mathrm{C}_{3}$

different configurations (a) and (b), see Fig. 2. In both configurations, $\boldsymbol{B}$ was in the basal plane $\left(\vartheta=90^{\circ}\right)$ while $\boldsymbol{B}_{1}$ was either parallel (a) or perpendicular (b) to this plane. In accordance with Eq. (8), the unit vector of $\boldsymbol{B}_{1}$ in the crystallographic frame is expressed as follows: in the case (a), $\psi=0^{\circ}$,

$$
l_{1}=\left(\begin{array}{c}
\sin \varphi \\
-\cos \varphi \\
0
\end{array}\right)
$$

and in the case (b), $\psi=90^{\circ}$,

$$
l_{1}=\left(\begin{array}{l}
0 \\
0 \\
1
\end{array}\right)
$$

Here, we focus on the dependence of intensities of certain EPR lines on the orientation of $\boldsymbol{B}_{1}$, described by the angle $\psi$, at a fixed orientation of $\boldsymbol{B}$. Figure 3 (left) shows experimental spectra recorded in both configurations. The dependence in question has been found to be particularly pronounced for two resonances (in fact, very closely spaced doublets) observed, respectively, at ca. 0.04 and $0.23 \mathrm{~T}$.

To get a closer insight in this issue, we have put forward a simulation code based on the spin Hamiltonian Eq. (10). The resonance fields have been calculated by diagonalizing the spin Hamiltonian matrix and considering all possible transitions between different energy levels. The intensities of different transitions have been calculated using Eq. (11) for the transition matrix element. A trial and error fitting procedure has been applied to obtain the following best-fit parameters (with error margins):

$$
\begin{array}{r}
g_{\|}=1.964 \pm 0.002 ; \quad g_{\perp}=2.018 \pm 0.002 ; \quad B_{2}^{0}=0.405 \pm 0.001 \mathrm{~cm}^{1} ; \\
g_{1}=-0.004 \pm 0.0005 ; \quad g_{2}=0.0409 \pm 0.002 ; \quad g_{3}=0.0409 \pm 0.002 ; \\
\quad g_{4}=0.0036 \pm 0.0002 ; \quad g_{5}=0.758 \pm 0.01 ; g_{6}=0.758 \pm 0.01 .
\end{array}
$$



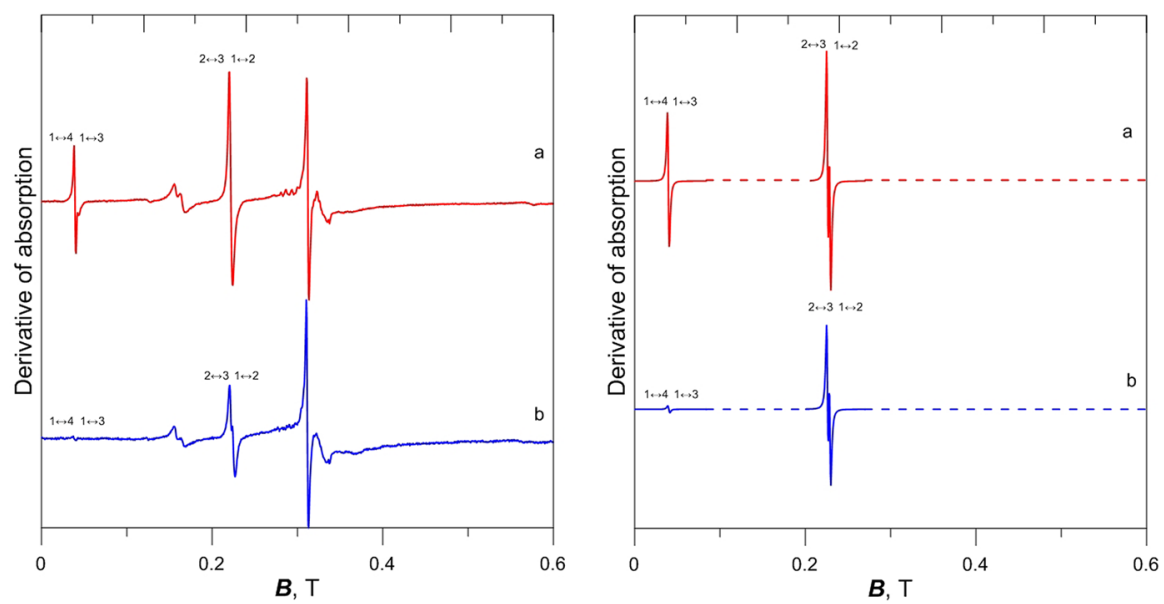

Fig. 3 Experimental (left) and computer generated (right) EPR spectra for $\boldsymbol{B}_{1}$ parallel, $\psi=0^{\circ}$ (curves a) and perpendicular, $\psi=90^{\circ}$ (curves $b$ ) to the basal plane of the crystal

One can see that in our case the $g$ tensor is not very anisotropic, and $g_{2}=g_{3}$ and $g_{5}=g_{6}$ because of the $\mathrm{Ni}^{3+}$ site symmetry.

Computer fittings to the two EPR features in question are shown in Fig. 3 (right). The exact values of the corresponding resonance fields have been determined as follows: 0.0396 and $0.0406 \mathrm{~T}$ for the low-field feature and 0.2259 and $0.2286 \mathrm{~T}$ for the medium-field feature.

Figure 4 shows calculated relative intensities of the above-mentioned resonances. The different Zeeman sublevels for $\mathrm{Ni}^{3+}$ are ordered from 1 to 4 . One can see that the transitions between adjacent levels $1 \leftrightarrow 2$ and $2 \leftrightarrow 3$ are globally much more intense than the transitions between non-adjacent levels $1 \leftrightarrow 3$ and $1 \leftrightarrow 4$, and the observed $\psi$-dependence of the line intensities is quite satisfactorily accounted for by the computer-generated spectra, as shown in Fig. 3.

Table 1 shows experimental and computed relative intensities of the chosen EPR transitions, taking for unity the intensity of the transition at $0.2259 \mathrm{~T}$. A certain discrepancy between experimental and computed intensities is certainly due to the difficulty of separating strongly superimposed doublet components in the experimental spectra.

\section{Conclusions}

In the EPR studies of Ni-doped $\mathrm{GaBO}_{3}$ single crystals, we have observed an unusually pronounced dependence of intensities of certain resonance lines on the orientation of microwave field $\boldsymbol{B}_{1}$ with respect to the crystallographic axes. Usually, in the analysis of the EPR spectra, the issue of relative intensities of different features is considered as secondary in comparison with that of the resonance fields. However, a reliable identification of various features in the experimental spectra requires careful computer simulations, in which case correct transition intensities 


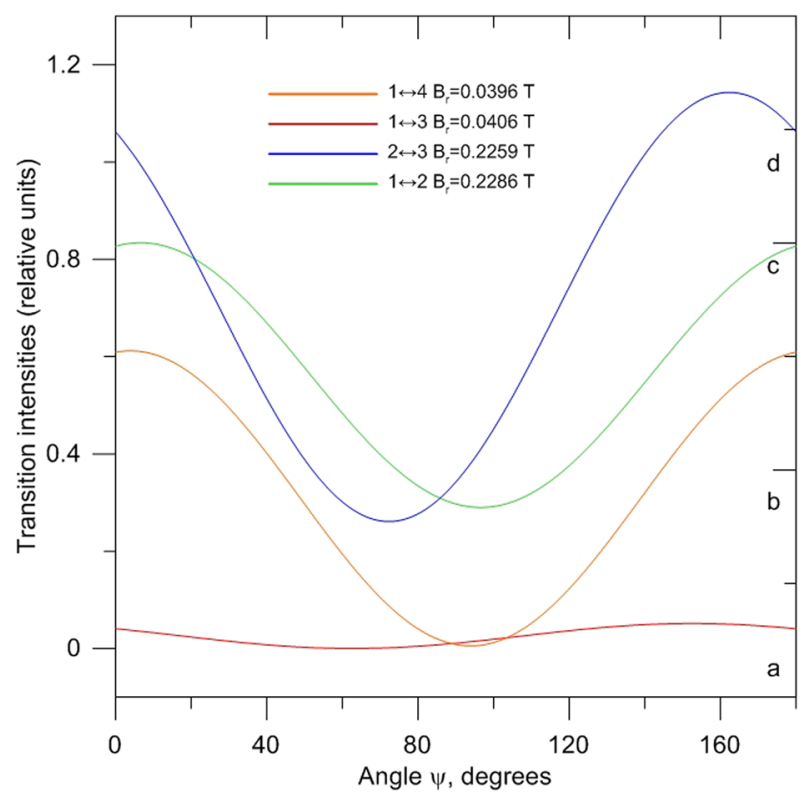

Fig. 4 Calculated dependence of the transition intensities on the angle $\psi$ for the low field $(a, b)$ and medium field $(c, d)$ doublets

Table 1 Relative intensities of the selected resonance lines

\begin{tabular}{|c|c|c|c|c|c|c|c|c|}
\hline \multirow{2}{*}{$\begin{array}{l}\text { Resonance field, T } \\
\text { Angle } \psi{ }^{\circ}\end{array}$} & \multicolumn{2}{|c|}{0.0396} & \multicolumn{2}{|c|}{0.0406} & \multicolumn{2}{|c|}{0.2259} & \multicolumn{2}{|c|}{0.2286} \\
\hline & 0 & 90 & 0 & 90 & 0 & 90 & 0 & 90 \\
\hline Intensity, experimental & 0.413 & 0.022 & 0.036 & 0.037 & 1 & 1 & 0.695 & 0.628 \\
\hline Intensity, calculated & 0.574 & 0.023 & 0.038 & 0.032 & 1 & 1 & 0.778 & 0.868 \\
\hline
\end{tabular}

become no less important facet than correct resonance fields. We have put forward a computer simulation code allowing to correctly account for both resonance fields and resonance intensities of different spectral features for different orientations of the magnetizing field and the microwave magnetic field. This procedure provides a good fitting to the experimental EPR spectra.

Acknowledgments This work was partially supported by RFBR Grant No. 144201557.

\section{References}

1. L.K. Aminov, I.N. Kurkin, S.P. Kurzin, I.A. Gromov, G.V. Mamin, R.M. Rakhmatullin, Phys. Solid State 49, 2086 (2007)

2. A. Abragam, B. Bleaney, Electron paramagnetic resonance of transition ions, General ed. by W. Marshall, D.H. Wilkinson (Clarendon Press, Oxford, 1970)

3. V.G. Grachëv, Zh. Eksp. Teor. Fiz. 92, 1834 (1987) 
4. J. Kliava, EPR Spectroscopy of Disordered Solids (Riga, Zinātne, 1988)

5. G. Korn, T. Korn, Mathematical Handbook for Scientists and Engineers, 2nd edn. (McGraw Hillbook, New York, 1968)

6. K. Seleznyova, M. Strugatsky, S. Yagupov, N. Postivey, A. Artemenko, J. Kliava, Phys. Status Solidi B 251, 1393 (2014)

7. S. Al'tshuler, B. Kozyrev, Electron paramagnetic resonance in compounds of transition elements, 2nd edn. (Wiley, New York Toronto Jerusalem London, 1974)

8. M. Strugatsky, S. Yagupov, N. Postivey, K. Seleznyova, E. Milyukova, V. Yagupov, Scientific Notes of TNU. Ser. Phys. Math. Sci. 24(63), 169 (2011)

9. S. Yagupov, E. Maksimova, I. Nayhatsky, V. Yagupov, E. Milyukova, K. Seleznyova, M. Strugatsky, in Abstracts of Internat. Conf. on Oxide Materials for Electronic Engineering OMEE 2014 (Lviv, Ukraine, 2014), p. 207 